\title{
Rotating compensator sampling for spectroscopic imaging ellipsometry
}

\author{
Y.H. Meng ${ }^{\mathrm{a}, \mathrm{b}}$, G. Jin ${ }^{\mathrm{a}, *}$ \\ a Institute of Mechanics, Chinese Academy of Sciences, 15, Bei-Si-Huan West Rd., Beijing 100190, PR China \\ ${ }^{\mathrm{b}}$ Graduate University, Chinese Academy of Sciences, 19A, Yuquan Rd., Beijing 100049, PR China
}

\section{A R T I C L E I N F O}

Available online 23 December 2010

\section{Keywords:}

Rotating compensator

Spectroscopic imaging ellipsometry

Spectroscopic ellipsometry

Imaging ellipsometry

Ellipsometry

Nanofilm pattern

\begin{abstract}
A B S T R A C T
In this work, a rotating compensator sampling for spectroscopic imaging ellipsometry (SIE) is presented and demonstrated by characterization of a $\mathrm{SiO}_{2}$ nanofilm pattern on $\mathrm{Si}$ substrate. Experiment results within spectrum of $400-700 \mathrm{~nm}$ show that the rotating compensator sampling is valid for SIE to obtain the ellipsometric angle distributions $\psi(x, y, \lambda)$ and $\Delta(x, y, \lambda)$ over the thin film pattern, the sampling times of $\psi(x, y)$ and $\Delta(x, y)$ with $576 \times 768$ pixels under each wavelength is less than $8 \mathrm{~s}$, the precision of fitting thickness of $\mathrm{SiO}_{2}$ is about $0.2 \mathrm{~nm}$ and the lateral resolution is $60.9 \mu \mathrm{m} \times 24.6 \mu \mathrm{m}$ in the parallel and perpendicular direction with respect to the incident plane.
\end{abstract}

(C) 2010 Elsevier B.V. All rights reserved.

\section{Introduction}

Ellipsometry has been developed into a powerful and versatile approach for the thin-film, especially for the nano-film [1-3]. It is based on detection of probe beam changes in polarization states before and after reflection upon sample surface and has the advantages of its remarkable non-contact and non-destructive advantages, sensitivity of atomic layer resolution and less limitation for specimen materials.

In order to satisfy the increasing demand in resolving capacities for characterization of the thin film, ellipsometry evolution has never ceased since the first ellipsometer founded in 1889 [4]. The single wavelength ellipsometry is commonly used in the routine task or realtime application to characterize the thin film with few unknown parameters. The spectroscopic ellipsometry (SE) with spectral resolving capability is widely used to characterize multi-layered thin film for obtaining the spectroscopic physical properties and structural properties [2,3]. However, intrinsically, the conventional ellipsometry is a single-point measurement method which limits its lateral resolution no less than the illuminated area by probe beam on the sample surface. Imaging ellipsometry, which combines high spatial resolution of optical microscopy with thin-film measurement capabilities of the ellipsometry, is developed to characterize the thin film pattern for physical and structural properties distribution [5-7]. In order to enhance the spectroscopic resolving capacity of imaging ellipsometry, spectroscopic imaging ellipsometry (SIE) is developed to characterize the spectroscopic, physical and spatial distribution for multi-layered nano-film patterns [8-12].

\footnotetext{
* Corresponding author. Tel./fax: +86 1082544138.

E-mail addresses: yhong@imech.ac.cn (Y.H. Meng), gajin@imech.ac.cn (G. Jin).
}

Sampling method plays vital role in SIE for quantitative measurement. SIE has the hybrid capabilities of ellipsometry, spectroscopy and microscopy, at a cost, SIE have to face the new challenges of them. Several sampling methods for ellipsometry have been well established including null $[5,13]$, rotating polarizing parts such as rotating polarizing, rotating analyzer $[14,15]$ or rotating compensator $[9,10,16]$, and the phase modulation sampling [17], but it is hardly to use them directly for SIE because of the available parts' limitation. Compared with conventional ellipsometry, the SIE has an expanded beam instead of a narrow one and 2 dimension camera instead of a single photo-detector such as photo-multiplier or semiconductor optoelectronic detector. The slow speed and high light intensity requirement makes the null sampling method is impractical to use in SIE. The intensity-detected dynamic range of the camera is much smaller than photo-detectors so that it is difficult to use the common-used rotating analyzer or polarizer sampling in SIE. The phase modulation is possible to use in the SIE by using of the synchronization of the coherent flash illumination or gated detector with the photo-elastic modulation to strobe measure images at specific times in modulation cycle but the system control is complicated.

In this work, a rotating compensator sampling for SIE is presented due to its available dynamic range fitting to the camera, as well as the non-ambiguous determination of ellipsometric angle $\Delta$, the insensitivity to light source and the detector polarization, the high accuracy even $\Delta$ near $0^{\circ}$ or $180^{\circ}$ and no need for a DC level, etc.

\section{Principle}

Here, the SIE with rotating sampling is based on polarizercompensator-specimen-analyzer (PCSA) configuration. A wide spectrum light source combined with a grating-scanning monochromator is used to provide a variable wavelength output for spectroscopic purpose. A collimating lens followed the monochromator exit 
converts the probe light into an expanded and parallel beam to illuminate a large area surface of a specimen at an angle of incidence (AOI). The PCSA sequence with rotating compensator sampling is used to detect the polarization change before and after the reflection upon the specimen surface. An achromatic lens is used to image the specimen onto a 2-dimension CCD camera for data acquisition.

Firstly, the rotating compensator sampling is carried out for quantitative measurement of the ellipsometric angles of $\psi(x, y)$ and $\Delta(x, y)$ distribution over a large area of specimen under a single wavelength. The azimuth of analyzer is preset at $+45^{\circ}$ or $-45^{\circ}$ and azimuth of polarizer is preset at an optimum angle with respect to the plane of incidence. Under the automatic control, the azimuth of compensator is changed with an equal angle interval step by step within an optical cycle $180^{\circ}$. At each sampling point of an azimuth of the compensator, an ellipsometric image is captured in grayscale, where the value in grayscale of each pixel $(x, y)$ corresponding to a micro area on the specimen takes the form

$$
\begin{aligned}
S_{n}(x, y)= & \alpha_{0}(x, y)+\alpha_{2 c}(x, y) \cos 2 C_{n}+\alpha_{2 s}(x, y) \sin 2 C_{n} \\
& +\alpha_{4 c}(x, y) \cos 4 C_{n}+\alpha_{4 s}(x, y) \sin 4 C_{n}
\end{aligned}
$$

where, $C_{n}$ is the azimuth of compensator of the sampling point. The coefficients $\alpha_{2 c} \sim \alpha_{4 s}$ relating to the properties of the specimen are obtained by Fourier analysis to all the images captured at all sampling point under present wavelength [18] as

$$
\begin{aligned}
& \alpha_{2 c}(x, y)=\frac{2}{m} \sum_{n}^{m-1} S_{n}(x, y) \cos 2 C_{n} \\
& \alpha_{2 s}(x, y)=\frac{2}{m} \sum_{n}^{m-1} S_{n}(x, y) \sin 2 C_{n} \\
& \alpha_{4 c}(x, y)=\frac{2}{m} \sum_{n}^{m-1} S_{n}(x, y) \cos 4 C_{n} \\
& \alpha_{4 s}(x, y)=\frac{2}{m} \sum_{n}^{m-1} S_{n}(x, y) \sin 4 C_{n}
\end{aligned}
$$

where $m$ is the number of sampling point. In order to deduce ellipsometric parameters properly, the total number of sampling points should be no less than 8 . Until now, the ellipsometric angles $\psi(x, y)$ and $\Delta(x, y)$ under present wavelength of each corresponding micro area on specimen can be achieved by [19]

$$
\tan 2 \Psi(x, y)=\frac{\sqrt{\left[\alpha_{2 s}(x, y)^{2}+\alpha_{2 c}(x, y)^{2}\right] \tan ^{2} \frac{\delta_{c}}{2}+4\left[\alpha_{4 s}(x, y) \cos 2 P-\alpha_{4 c}(x, y) \sin 2 P\right]^{2}}}{-2\left[\alpha_{4 c}(x, y) \cos 2 P+\alpha_{4 s}(x, y) \sin 2 P\right]}
$$

$\tan \Delta(x, y)=\frac{1}{2} \tan \left(\frac{\delta_{C}}{2}\right) \frac{\alpha_{2 c}(x, y) \sin 2 P-\alpha_{2 s}(x, y) \cos 2 P}{\alpha_{4 c}(x, y) \sin 2 P-\alpha_{4 s}(x, y) \cos 2 P}$

where $\delta_{C}$ is the phase retardation of the compensator between fast and slow axes, $P$ is the azimuths of the polarizer. The spectroscopic ellipsometric angles $\psi(x, y, \lambda)$ and $\Delta(x, y, \lambda)$ distribution within a spectrum is obtained by scanning the wavelength.

In order to cancel out misalignments and other internal system artifacts, the two-zone averaging technique for is used [19], which means to average the ellipsometric angles $\psi\left(x, y, \lambda, A=45^{\circ}\right)$ and $\Delta\left(x, y, \lambda, A=-45^{\circ}\right)$ sampled under the azimuth of the analyzer is preset at $+45^{\circ}$ or $-45^{\circ}$, respectively. Further, azimuth of the polarizer is preset at an optimum angle with respect to the incident plane to reduce systematic error. The multi-image averaging method is used to reduce the random noise.

During the spectroscopic measurement, the detected signal in each pixel of the image is variable with the wavelength scanning due to some factors including the dispersive properties of the specimen, the intensity spectrum of light source, the transmission spectrum of the polarizer, analyzer and compensator, as well as the spectrum response of the 2-Dimension camera. A variable expose time of CCD camera is used to improve the signal-to-noise ratio in spectroscopic measurement to reduce this effect of spectrum. Firstly, we get the relative intensity spectrum coefficient $C_{\lambda}$ of the SIE system for the tested sample. Then, at each sampling point of azimuth of compensator the system acquires $M$ images and averaged them with the weighed coefficient $\mathrm{M}^{*} \mathrm{C}_{\lambda}$ pixel by pixel. By this method, both the sensitivity and ratio of signal to noise are improved.

\section{Experiment setup}

The SIE with rotating compensator sampling is shown in Fig. 1. A $150 \mathrm{~W}$ xenon short arc lamp (OSRAM, Germany) powered by a supply (Newport, U.S.) with light ripple less than $0.5 \%$ regulation accuracy is used to provide a stable outgoing flux with continuous spectrum from UV to IR. In order to adequately utilize the light flux, an achromatic convergent lens set with a focal length of $200 \mathrm{~mm}$ and a diameter of $50 \mathrm{~mm}$ is used to converge the light beam into the entrance slit of a mechanical scanning grating monochromator (7-Star, China) with a focal length of $150 \mathrm{~mm}$ and an F-number of $1 / 4.5$. The monochromator, under the control of the computer, provides a spectrum of 260-1600 nm with adjustable entrance and exit for control of the light intensity and bandwidth, respectively. A glass optical fiber bound (Chunhui, China) by a spectrum of $380-1300 \mathrm{~nm}$ and a diameter of $1.0 \mathrm{~mm}$ is used to flexibly transfer the probe light from the exit of the monochromator to the focus point of the second achromatic convergent lens set. This collimates the single wavelength into an expanded probe beam with a diameter of $25 \mathrm{~mm}$ for thin film detection at the same angle of incidence.

Two Glan-Taylor prisms (Melles Griot, USA) with a spectral range of $250-2300 \mathrm{~nm}$ and an extinction ratio of $10^{-5}$ are used as the polarizer and the analyzer, respectively. A mica retardation plate (Melles Griot, USA) with a spectral range of $400-700 \mathrm{~nm}$ is used as a compensator. Its phase retardation between fast axis and slow axis is near $90^{\circ}$, which is calibrated by a commercial V-VASE ellipsometer (J. A. Woollam, USA) in transmission mode and fitted by the Cauchy model. In order to adjust the azimuth with respect to the plane of incidence, the polarizer, compensator, and analyzer are fixed in hollow annular rotation stages driven by a servo motor (Newport, US) with a maximum speed of $40^{\circ}$ per second and an absolute accuracy of $0.015^{\circ}$. The azimuths of the polarizing parts are calibrated by a self-calibrating method. The specimen is fixed in the specimen stage by a vacuum pump with a linear-motor-driven free piston (Nitto Kohki, Japan) and aligned by an optical self-collimated telescope (Micro-Radian, US).

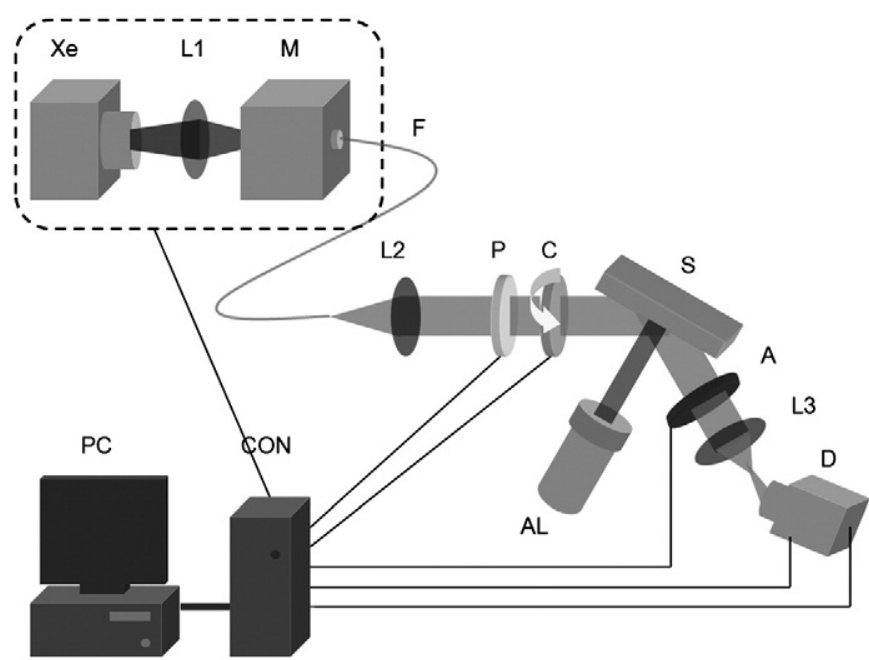

Fig. 1. The schematic diagram of SIE with rotating compensator sampling. Xe - Xe lamp; L1 and L2 - collimating lens; $\mathrm{M}$ - monochromator; $\mathrm{F}$ - optical fiber; $\mathrm{P}$ - polarizer; $\mathrm{C}$ compensator; S - specimen; A - analyzer; L3 - imaging lens; D - CCD camera; AL alignment telescope; CON - controller; and PC - industry computer. 


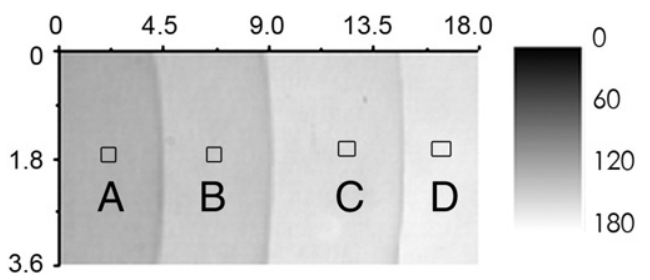

Fig. 2. Raw image in grayscale with size of $300 \times 150$ pixels captured at $\mathrm{AOI}=70^{\circ}$, $\lambda=500 \mathrm{~nm}, \mathrm{P}=0^{\circ}, \mathrm{A}=45^{\circ}$, and $\mathrm{C}=56.25^{\circ}$. The rectangles denoted by $\mathrm{A}, \mathrm{B}, \mathrm{C}$ and $\mathrm{D}$ are selected area with top-left positions $(x, y)$ in pixel are $A(1.83 \mathrm{~mm}, 1.82 \mathrm{~mm}), \mathrm{B}(6.70 \mathrm{~mm}$, $1.79 \mathrm{~mm}), \mathrm{C}(12.24 \mathrm{~mm}, 1.57 \mathrm{~mm})$ and $\mathrm{D}(17.24 \mathrm{~mm}, 1.40 \mathrm{~mm})$, respectively. Each area consists of $12 \times 12$ pixels and their corresponding area in film pattern is $0.731 \mathrm{~mm}$ $(\mathrm{H}) \times 0.295 \mathrm{~mm}(\mathrm{~V})$.

An achromatic imaging lens set (Zeiss, Germany) with a focal length of $50 \mathrm{~mm}$ and an $\mathrm{f}$-number of 1.4 is used to image the specimen onto a scientific B/W video CCD camera (SONY, Japan). The camera features a corresponding spectrum range of $400-900 \mathrm{~nm}$, sensitive area of $768 \times 576$ pixels with size of $6.0 \mathrm{~mm} \times 4.96 \mathrm{~mm}$, and single pixel size of $6.5 \mu \mathrm{m} \times 6.25 \mu \mathrm{m}$. The electronic gain is fixed for quantitative photonic intensity measurement. The sampling speed is $30 \mathrm{fps}$ at maximum and $12 \mathrm{fps}$ at normal operation including the process of image capturing and storing. The nonlinearity of the CCD camera is better than $1 \%$. The image signal is inputted into an image grab board (Matrox, Canada) inserted in computer for analog-todigital (A/D) conversion and digital image processing. The ellipsometric images are recorded in 8-bit (0-255) grayscale format. Image averaging method is used to improve the signal-to-noise ratio [20].

The SIE system is operated under an automatic control system including operation system, control hardware and duty functions [10]. The operation system includes the motion control, image acquisition and data analysis. By using of the control hardware consisting of interface card, motor driver and feedback receiver, the automatic functions are operated. The major motion functions include: system initialization such as specimen fixing and self-calibrating; system setting such as azimuths settings of polarizer, compensator and analyzer; wavelength scanning of the monochromator for wavelength selection; the light intensity adjusting for to match the probe beam intensity with the dynamic range of the CCD camera; auto-focusing to image the whole specimen clearly on to the CCD sensing surface; angle of incidence adjustment to improve the detection sensitivity or to obtain much data for fitting; and moving parts positioning for the feedback of the location of each moving part.

The major image acquisition function is the camera image sampling, which includes the image capture upon the requirement such as the number of frame $m$, the time interval and the average of multi-image, etc.; and image process such as the choice of an interested special area, the readout of the value in grayscale in the area, the image transfer to 3D, etc.

The major data analysis functions under a software that mainly include: Fourier analysis to obtain coefficients $\alpha_{2 c} \sim \alpha_{4 s}$ in Eq.(2); ellipsometric angles $\Psi(x, y, \lambda)$ and $\Delta(x, y, \lambda)$ calculation of each micro-area of the specimen corresponding to pixel of image according Eq. (3), and the deduction for properties of the specimen such as the thickness and distribution of the thin film, the refraction index.

\section{Experiment results}

Here, we use a stepped $\mathrm{SiO}_{2}$ layer on $\mathrm{Si}$ substrate as specimen to demonstrate the performance of the sampling method for SIE. It is prepared by eroding the $\mathrm{SiO}_{2}$ layer with a thickness of $65 \mathrm{~nm}$ on Si with hydrofluoric acid under different controlled time. The specimen has size of $18.27(\mathrm{H}) \mathrm{mm} \times 3.69(\mathrm{~V}) \mathrm{mm}$, which consisting of four $\mathrm{SiO}_{2}$ thin film layers with the different thicknesses. As a reference, a single point variable angle spectroscopic ellipsometer (VASE, by J.A. Woollam Co.) is used to characterize the thickness of the stepped layers, with the physical model of air- $\mathrm{SiO}_{2}-\mathrm{Si}$ where the fitting thickness of the four layers is about $8 \mathrm{~nm}, 15 \mathrm{~nm}, 25 \mathrm{~nm}$, and $35 \mathrm{~nm}$, respectively.

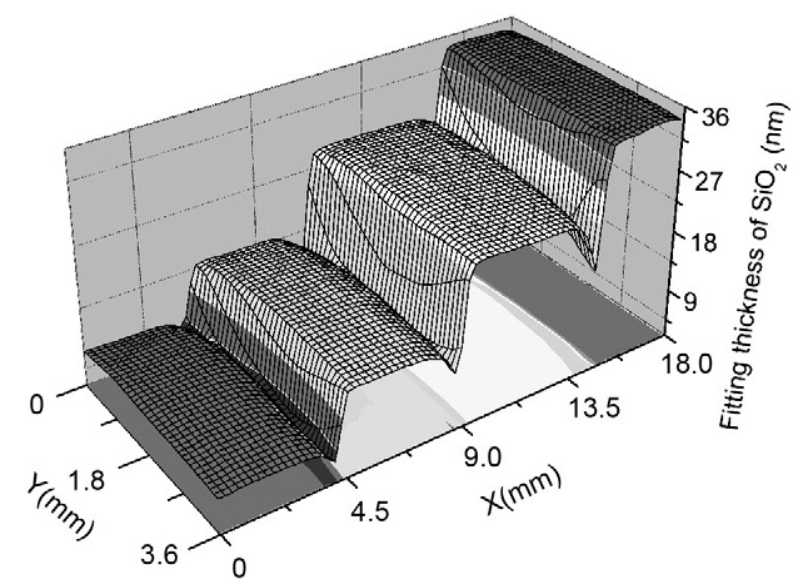

Fig. 3. The fitting thickness of $\mathrm{SiO}_{2}$ stepped layer on $\mathrm{Si}$ substrate by using the physical model of $\mathrm{Air}-\mathrm{SiO}_{2}-\mathrm{Si}$.

The angle of incidence is set at $70^{\circ}$ near the Brewster's angle of the silicon substrate. The optical magnification is 0.254 and the specimen size image is $300(\mathrm{H}) \times 150(\mathrm{~V})$ pixels with the corresponding lateral resolution of $60.9(\mathrm{H}) \times 24.6(\mathrm{~V})(\mu \mathrm{m})$ in the parallel and perpendicular direction with respect to the incident plane. The spectral range of $400-700 \mathrm{~nm}$ is chosen and the wavelength interval is set at $50 \mathrm{~nm}$.

Fig. 2 shows the raw image in grayscale recorded under the condition of $\lambda=500 \mathrm{~nm}, \mathrm{P}=0^{\circ}, \mathrm{A}=45^{\circ}$, and $\mathrm{C}=56.25^{\circ}$. The corresponding ellipsometric angles image $\psi(x, y, 500 \mathrm{~nm})$ and $\Delta(x, y, 500 \mathrm{~nm})$ can be obtained by Eqs. (2) and (3) [10].

By using the wavelength scanning, the SIE obtains the spectral ellipsometric parameters $\psi(x, y, \lambda)$ and $\Delta(x, y, \lambda)$ images within the spectrum of 400-700 $\mathrm{nm}$. It is hardly to show the 4-dimension of $\psi(x$, $y, \lambda)$ and $\Delta(x, y, \lambda)$ image in 3-dimension space. We can easily show the spectroscopic ellipsometric angles image $\psi(x, y, \lambda)$ and $\Delta(x, y, \lambda)$ of one selected row in 3-dimension [10]. At present SIE setup, the sampling speed of $\psi(x, y, \lambda)$ and $\Delta(x, y, \lambda)$ with $768 \times 576$ pixels is $8 \mathrm{~s}$ per wavelength, where the data acquisition time of the CCD camera is less than $0.8 \mathrm{~s}$, and the time of mechanical component movements is less than $7.2 \mathrm{~s}$.

In order to evaluation the thickness distribution of the $\mathrm{SiO}_{2}$, we use the physical model of air- $\mathrm{SiO}_{2}-\mathrm{Si}$. The fitting procedure is operated by the use of the WVASE 32 (J.A.Woollam). The fitting thickness distribution over the whole area is shown in Fig. 3. It can be seen that the thickness distribution varies slightly within each layer and changes abruptly between two adjoining layers. The abnormal jump down in the boundaries between two adjoining layers mainly results from the sample preparation procedure when hydrofluoric acid solution sways and makes the boundaries rough and irregular. In order to evaluate the precision of the rotating compensator sampling, we select four areas marked as A, B, C, and D, respectively. Correspondingly, their top-left positions $(x, y)$ is A $(1.83 \mathrm{~mm}, 1.82 \mathrm{~mm}), \mathrm{B}(6.70 \mathrm{~mm}, 1.79 \mathrm{~mm}), \mathrm{C}$ $(12.24 \mathrm{~mm}, 1.57 \mathrm{~mm})$ and $\mathrm{D}(17.24 \mathrm{~mm}, 1.40 \mathrm{~mm})$, respectively. Each area consists of $12 \times 12$ pixels and their corresponding area in film pattern is $0.731(\mathrm{H}) \times 0.295(\mathrm{~V})(\mathrm{mm})$. The experimental data $\psi(\lambda)$ and $\Delta(\lambda)$ of each selected area A, B, C and D is the average value of the $12 \times 12$ sub-areas. Table 1 shows the fitting result of selected area. The thickness of $\mathrm{SiO}_{2}$ film at selected areas of $\mathrm{A}, \mathrm{B}, \mathrm{C}$ and $\mathrm{D}$ is $8.18 \mathrm{~nm}$, $15.5 \mathrm{~nm}, 25.3 \mathrm{~nm}$, and 34.6, respectively, which is almost same as the

Table 1

Fitting thickness of $\mathrm{SiO}_{2}$ on selected area by use of the software WVASE32 (J.A.Woollam, U.S.) and the physical model Air- $\mathrm{SiO}_{2}-\mathrm{Si}$.

\begin{tabular}{lllll}
\hline Selected area & A & B & C & D \\
\hline Fitting thickness $(\mathrm{nm})$ & $8.18 \pm 0.09$ & $15.5 \pm 0.1$ & $25.3 \pm 0.2$ & $34.6 \pm 0.2$ \\
MSE & 1.040 & 0.846 & 1.382 & 1.854 \\
\hline
\end{tabular}


measured result by the VASE ellipsometer. The thickness deviation shows the fitting thickness precision, it is smaller with the decrease of film thickness. The minimum square error (MSE) function is used to evaluate the fitting degree of calculated data and the experimental data [9].

\section{Conclusion}

The rotating compensator sampling for an automatic SIE is presented and is demonstrated within spectrum $400-700 \mathrm{~nm}$ by characterization of $\mathrm{S} \mathrm{SiO}_{2}$ stepped layer pattern on $\mathrm{Si}$ substrate. The rotating compensator sampling can be used for SIE to qualitatively obtain the spectroscopic ellipsometric angles of $\psi(x, y, \lambda)$ and $\Delta(x, y$, $\lambda$ ) distribution of the thin film pattern. At present setup, the sampling speed of $\psi(x, y)$ and $\Delta(x, y)$ with $768 \times 576$ pixels is $8 \mathrm{~s}$ under a single wavelength, where the data acquisition time of the CCD camera is less than $0.8 \mathrm{~s}$, and the time of mechanical component movements is less than $7.2 \mathrm{~s}$. Further fitting analysis by the use of the model air-SiO ${ }_{2}-\mathrm{Si}$ indicates that the thickness precision is approximately $0.2 \mathrm{~nm}$. By the use of the two-zero averaging, the accuracy of the SIE is nearly same as the conventional spectroscopic ellipsometer, while the measurement time will be about $19 \mathrm{~s}$. Furthermore, the SIE performance can be improved by optimizing the system parameters [21-23].

However, there are still some problems in carrying out the rotating compensator sampling for SIE. The ratio of signal to noise is much lower than that of rotating analyzer or polarizer sampling widely used in conventional ellipsometry since a narrow intensity-detected dynamic range of the 2-dimension camera resulting from potential well depth. The response speed of camera is slower more than three orders than the conventional photo-detector, which makes it difficult to improve the measurement speed and hardly to use in fast dynamic process monitoring.

\section{Acknowledgement}

The Chinese Academy of Sciences YZ0629, KJCX2-YW-M03 and KJCX2-YW-M04; the National Basic Research Program of China 2009CB320300; and the National High Technology Research and Development Program (863) of China 2008AA02Z419 are acknowledged for their financial supports.

\section{References}

[1] R.M.A. Azzam, N.M. Bashara, Ellipsometry and Polarized Light, North-Holland Publishing Co., Amsterdam, 1977.

[2] H.G. Tompkins, E.A. Irene, Handbook of Ellipsometry, William Andrew Inc., New York, 2005.

[3] H. Fujiwara, Spectroscopic Ellipsometry: Principles and Application, John Wiley \& Sons Ltd., England, 2009.

[4] P. Drude, Ann. Phys. Chem. 36 (1889) 532.

[5] T. Mishima, K.C. Kao, Opt. Eng. 21 (1982) 1074.

[6] G. Jin, R. Jansson, H. Arwin, Rev. Sci. Instrum. 67 (1996) 2930-2936.

[7] L. Asinovski, D. Beaglehole, M.T. Clarkson, Phys. Status Solidi A 205 (2008) 764

[8] A.J. Choi, T.J. Kim, Y.D. Kim, J.H. Oh, J. Jang, J. Korean Phys. Soc. 48 (2006) 1544.

[9] Y.H. Meng, S. Chen, G. Jin, Chin. Opt. Lett 8 (2010) 114, Supplement.

[10] Y.H. Meng, Y.Y. Chen, C. Qi, L. Liu, G. Jin, Phys. Status Solidi C 5 (2008) 1050.

[11] M. Vaupel, S. Yunfeng, Y. Zhimin, Phys. Status Solidi A 205 (2008) 772.

[12] M. Vaupel, M. Vinnichenko, Phys. Status Solidi C 5 (2008) 1137.

[13] M. Harke, M. Stelzle, H.R. Motschmann, Thin Solid Films 284-285 (1996) 412.

[14] R.F. Cohn, J.W. Wagner, J. Kruger, Appl. Opt. 27 (1988) 4664.

[15] A. Albersdo rfer, G. Elender, G. Mathe, K.R. Neumaier, P. Paduschek, E. Sackmann, Appl. Phys. Lett. 72 (1998) 2930.

[16] D. Beaglehole, Rev. Sci. Instrum. 59 (1988) 2557.

[17] C.Y. Han, Y.F. Chao, Rev. Sci. Instrum. 77 (2006) 023107.

[18] P.S. Hauge, F.H. Dill, Opt. Commun. 14 (1975) 431.

[19] R. Kleim, L. Kuntzler, A. Elghemmaz, J. Opt. Soc. Am. A 11 (1994) 2550

[20] Y. Meng, G. Jin, Opt. Prec. Eng. 8 (2000) 316, (in Chinese)

[21] D.S. Sabatke, M.R. Descour, E.L. Dereniak, W.C. Sweatt, S.A. Kemme, G.S. Phipps, Opt. Lett. 25 (2000) 802.

[22] J. Scott Tyo, Appl. Opt. 41 (2002) 619.

[23] E. Garcia-Caurel, A. D. Martino and B. Drevillon, U.S. Patent No. 2007/0146706 A1, Jun. 28, 2007. 